\title{
ENERGY ESTIMATES FOR THE BIHARMONIC EQUATION IN THREE DIMENSIONS
}

\author{
By \\ CHANGHAO LIN \\ South China Normal University, Guangzhou, People's Republic of China
}

1. Introduction. In this paper, we are concerned with energy decay estimates for solutions of the biharmonic equation in a semi-infinite cylinder with nonzero boundary conditions on the finite end. Decay estimates for various energies are derived and compared.

Saint-Venant's principle in the context of plane elastostatics has been investigated by many authors [1-5]. Various types of arguments are employed to establish energy decay estimates for the resulting biharmonic equation in a semifinite strip. It is, however, not yet known how to employ a simple technique to yield a sharp decay rate-one which approximates the exact decay rate and makes effective application of Saint-Venant's principle possible.

Although the analogous three-dimensional biharmonic problem does not have the same elasticity interpretation as its counterpart in the plane, it is nevertheless of mathematical interest to try to extend the decay results in $\mathbb{R}^{2}$ to analogous results in $\mathbb{R}^{3}$. In this paper, we derive and compare decay estimates using both direct analogues of known arguments for the two-dimensional case and new techniques. In $\S 3$ we derive exponential decay estimates for the first-order energy and the second-order energy. Then we establish an alternate energy decay estimate in $\S 4$. An equivalence between the first-order energy and the second-order energy is derived in $\S 5$. Finally, we establish upper bounds for the total energies in terms of the given boundary data on the finite end. As we shall see, although there is an obvious similarity between the plane biharmonic equation and the spatial biharmonic equation, our arguments in this paper are quite different from those of [1-3]. Meanwhile, the techniques and results of this paper could be extended to the case of arbitrary dimension.

2. Preliminary results. Let $R$ be the interior of a semi-infinite cylinder whose cross section $D$ is bounded by one or more piecewise smooth simple closed curves. Choose Cartesian coordinates $x_{1}, x_{2}$, and $x_{3}$ with the origin as one end of the cylinder and the $x_{3}$-axis parallel to the generator. Let $\phi$ be a smooth solution of the biharmonic equation

$$
\Delta \Delta \phi=0 \quad \text { on } R=D \times[0, \infty)
$$

Received March 30, 1992.

1991 Mathematics Subject Classification. Primary 35B40, 35B45, 73C50.

(C)1994 Brown University 
subject to the boundary conditions

$$
\begin{gathered}
\phi\left(x_{1}, x_{2}, 0\right)=f\left(x_{1}, x_{2}\right), \quad\left(x_{1}, x_{2}\right) \in D ; \\
\phi_{, 3}\left(x_{1}, x_{2}, 0\right)=g\left(x_{1}, x_{2}\right), \quad\left(x_{1}, x_{2}\right) \in D ; \\
\phi\left(x_{1}, x_{2}, x_{3}\right)=\frac{\partial \phi}{\partial n}=0 \quad \text { on } \partial D ; \\
\left.\phi_{, 3,} \phi_{, i j} \rightarrow 0 \quad \text { (uniformly in }\left(x_{1}, x_{2}\right) \in D\right) \text { as } x_{3} \rightarrow \infty
\end{gathered}
$$

where $f$ and $g$ are prescribed functions satisfying

$$
f=g=\frac{\partial f}{\partial n}=\frac{\partial g}{\partial n}=0 \text { on } \partial D
$$

and $\partial / \partial n$ represents the outward normal derivative at points of the boundary $\partial D$. Throughout, subscripts repeated with Latin symbols have the range $1,2,3$ and with Greek symbols the range 1,2 ; a comma denotes partial differentiation:

$$
\phi_{, j}=\frac{\partial \phi}{\partial x_{j}} \text {. }
$$

In what follows, we shall assume that a solution of the system (2.1)-(2.5) which is four times continuously differentiable on the closure $\bar{R}$ of $R$ exists. The point sets $\left\{\left(x_{1}, x_{2}, x_{3}\right) \mid\left(x_{1}, x_{2}, x_{3}\right) \in R, x_{3}>z \geq 0\right\}$ are denoted by $R_{z}$, and so $R_{0} \equiv R$. For each fixed $z>0, D_{z}$ denotes the cross section of $R$ containing the points $\left(x_{1}, x_{2}, z\right)$.

Before proceeding to establish the main results, it is convenient to assemble here some auxiliary results.

Lemma 2.1. Let $\phi$ be a solution of the system $(2.1)-(2.5)$; then $\left.D_{x} \phi\right|_{\partial D}=0$, where $D_{x} \phi$ denotes the gradient of $\phi$ with respect to $\left(x_{1}, x_{2}\right)$.

LEMMA 2.2 (Conservation Law). For any solution of the system (2.1)-(2.5), we have

$$
\int_{D_{x}}\left[\phi_{, 3} \phi_{, 333}-\frac{1}{2} \phi_{, 33}^{2}+\frac{1}{2} \phi_{, \alpha \beta} \phi_{, \alpha \beta}-\phi_{, \alpha 3} \phi_{, \alpha 3}\right] d A=\text { const. }
$$

Furthermore, if we assume that $\phi_{, 333}=O(1)$, as $x_{3} \rightarrow \infty$, then

$$
\int_{D_{x}}\left[\phi_{, 3} \phi_{, 333}-\frac{1}{2} \phi_{, 33}^{2}+\frac{1}{2} \phi_{, \alpha \beta} \phi_{, \alpha \beta}-\phi_{, \alpha 3} \phi_{, \alpha 3}\right] d A=0 .
$$

Lemma 2.1 is obvious, and Lemma 2.2 is the direct analogue of the two-dimensional result (see, e.g., Horgan [3]).

Now, let us recall some Poincaré inequalities required in subsequent calculation.

(i) If $\varphi\left(x_{1}, x_{2}\right) \in C^{\prime}(D),\left.\varphi\right|_{\partial D}=0$, then

$$
\int_{D} \varphi^{2} d x_{1} d x_{2} \leq \frac{1}{\lambda_{1}} \int_{D} \varphi_{, \alpha} \varphi_{, \alpha} d x_{1} d x_{2}
$$

where $\lambda_{1}$ is the first eigenvalue in the two-dimensional fixed membrane problem. A lower bound for $\lambda_{1}$ is given by the Faber-Krahn inequality [8]

$$
\lambda_{1} \geq \pi j_{0}^{2} /|D|
$$


where $j_{0}$ is the smallest positive zero of the Bessel function $J_{0}(y)\left(j_{0} \doteq 5.783\right)$ and $|D|$ represents the area of domain $D$.

(ii) If $\varphi\left(x_{1}, x_{2}\right) \in C^{2}(D), \varphi=D \varphi=0$ on $\partial D$, then

$$
\int_{D} \varphi_{, \alpha} \varphi_{, \alpha} d x_{1} d x_{2} \leq \frac{1}{\Lambda} \int_{D} \varphi_{, \alpha \beta} \varphi_{, \alpha \beta} d x_{1} d x_{2} .
$$

Here $\Lambda \geq \lambda_{2}$, where $\lambda_{2}$ is the second eigenvalue in the fixed membrane problem [9].

(iii) If $\varphi\left(x_{1}, x_{2}\right)$ satisfies the same conditions as in (ii), then

$$
\int_{D} \varphi^{2} d x_{1} d x_{2} \leq \frac{1}{\Omega} \int_{D} \varphi_{, \alpha \beta} \varphi_{, \alpha \beta} d x_{1} d x_{2}
$$

where $\Omega \geq \Lambda \lambda_{1} \geq \lambda_{2} \lambda_{1}$ [9].

3. Exponential decay estimates for the $E_{1}(z)$ and $E_{2}(z)$. We define

$$
\begin{aligned}
& E_{1}(z)=\int_{R_{z}} \phi_{, i j} \phi_{, i j} d V, \quad z \geq 0, \\
& E_{2}(z)=\int_{R_{z}} \phi_{, i j 3} \phi_{, i j 3} d V, \quad z \geq 0 .
\end{aligned}
$$

We call $E_{1}(z)$ the first-order energy and $E_{2}(z)$ a second-order energy. We first derive exponential decay for $E_{2}(z)$; then we show that $E_{1}(z)$ has the same decay rate as $E_{2}(z)$.

It is clear that

$$
\begin{aligned}
E_{2}(z) & =\int_{R_{z}}\left(\phi_{, i j 3} \phi_{, i 3}\right)_{, j} d V-\int_{R_{z}} \phi_{, i j j 3} \phi_{, i 3} d V \\
& =-\int_{D_{z}} \phi_{, i 3} \phi_{, i 33} d A-\int_{R_{z}}\left(\phi_{, i j j 3} \phi_{, 3}\right)_{, i} d V \\
& =-\frac{1}{2} \int_{D_{z}}\left(\phi_{, i 3} \phi_{, i 3}\right)_{, 3} d A+\int_{D_{z}} \phi_{, 3} \phi_{, j j 33} d A \\
& =-\frac{1}{2} \frac{d}{d z} \int_{D_{z}}\left(\phi_{, \alpha \beta} \phi_{, \alpha \beta}+\phi_{, 33}^{2}\right) d A
\end{aligned}
$$

and

$$
E_{2}^{\prime}(z)=-\int_{D_{z}} \phi_{, i j 3} \phi_{, i j 3} d A
$$

We now establish

THEOREM 3.1. Let $\phi$ be a solution of the system $(2.1)-(2.5)$; then $E_{2}(z), E_{1}(z)$ satisfy the inequalities

$$
\begin{aligned}
& E_{2}(z) \leq E_{2}(0) e^{-0.66 \sqrt{\lambda_{1}} z}, \quad z \geq 0, \\
& E_{1}(z) \leq \frac{9.17}{\lambda_{1}} E_{2}(0) e^{-0.66 \sqrt{\lambda_{1}} z}, \quad z \geq 0 .
\end{aligned}
$$


Proof. Let $K$ be an as yet undetermined positive constant. Upon using Eqs. (3.3), (3.4), and the arithmetic geometric mean inequality, we get

$$
\begin{aligned}
E_{2}(z)+K \lambda_{1}^{-1 / 2} E_{2}^{\prime}(z) \leq & \frac{\varepsilon \lambda_{1}^{1 / 2}}{2} \int_{D_{z}} \phi_{, \alpha \beta} \phi_{, \alpha \beta} d A \\
& +\frac{\lambda_{1}^{-1 / 2}}{2 \varepsilon} \int_{D_{z}} \phi_{, \alpha \beta 3} \phi_{, \alpha \beta 3} d A \\
& -\int_{D_{z}} \phi_{, 33} \phi_{, 333} d A-K \lambda_{1}^{-1 / 2} \int_{D_{z}} \phi_{, \alpha \beta 3} \phi_{, \alpha \beta 3} d A \\
& -2 K \lambda_{1}^{-1 / 2} \int_{D_{z}} \phi_{, \alpha 33} \phi_{, \alpha 33} d A-K \lambda_{1}^{-1 / 2} \int_{D_{z}} \phi_{, 333}^{2} d A .
\end{aligned}
$$

Applying the Conservation Law, we obtain

$$
\begin{aligned}
& \frac{\varepsilon \lambda_{1}^{1 / 2}}{2} \int_{D_{z}} \phi_{, \alpha \beta} \phi_{, \alpha \beta} d A \\
& \quad=-\varepsilon \lambda_{1}^{1 / 2} \int_{D_{z}} \phi_{, 3} \phi_{, 333} d A+\frac{\varepsilon \lambda_{1}^{1 / 2}}{2} \int_{D_{z}} \phi_{, 33}^{2} d A+\varepsilon \lambda_{1}^{1 / 2} \int_{D_{z}} \phi_{, \alpha 3} \phi_{, \alpha 3} d A .
\end{aligned}
$$

Making use of the arithmetic geometric mean inequality once again, we get

$$
\begin{gathered}
-\varepsilon \lambda_{1}^{1 / 2} \int_{D_{z}} \phi_{, 3} \phi_{, 333} d A \leq \varepsilon \lambda_{1}^{1 / 2}\left[\frac{\beta^{-1} \lambda_{1}}{2} \int_{D_{z}} \phi_{, 3}^{2} d A+\frac{\beta \lambda_{1}^{-1}}{2} \int_{D_{z}} \phi_{, 333}^{2} d A\right], \\
-\int_{D_{z}} \phi_{, 33} \phi_{, 333} d A \leq \frac{\lambda_{1}^{1 / 2}}{2 \gamma} \int_{D_{z}} \phi_{, 33}^{2} d A+\frac{\gamma \lambda_{1}^{-1 / 2}}{2} \int_{D_{z}} \phi_{, 333}^{2} d A .
\end{gathered}
$$

Then, using inequalities (2.9)-(2.11), we get

$$
\begin{aligned}
E_{2}(z)+K \lambda_{1}^{-1 / 2} E_{2}^{\prime}(z) \leq & \lambda_{1}^{-1 / 2}\left(-K+\frac{\gamma}{2}+\frac{\varepsilon \beta}{2}\right) \int_{D_{z}} \phi_{, 333}^{2} d A \\
& +\lambda_{1}^{-1 / 2}\left(-2 K+\frac{1}{2 \gamma}+\frac{\varepsilon}{2}\right) \int_{D_{z}} \phi_{, \alpha 33} \phi_{, \alpha 33} d A \\
& +\lambda_{1}^{-1 / 2}\left(-K+\frac{\varepsilon \lambda_{1}^{2}}{2 \beta \Omega}+\frac{1}{2 \varepsilon}+\frac{\varepsilon \lambda_{1}}{\Lambda}\right) \int_{D_{z}} \phi_{, \alpha \beta 3} \phi_{, \alpha \beta 3} d A .
\end{aligned}
$$

Now, we choose appropriate positive constants $\varepsilon, \beta, \gamma$, and $K$ such that

$$
\begin{gathered}
-K+\frac{\gamma}{2}+\frac{\varepsilon \beta}{2} \leq 0, \\
-2 K+\frac{1}{2 \gamma}+\frac{\varepsilon}{2} \leq 0, \\
-K+\frac{\varepsilon \lambda_{1}^{2}}{2 \beta \Omega}+\frac{1}{2 \varepsilon}+\frac{\varepsilon \lambda_{1}}{\Lambda} \leq 0 .
\end{gathered}
$$


Since $\Omega \geq \Lambda \lambda_{1} \geq \lambda_{1}^{2}, \Lambda \geq \lambda_{2}>\lambda_{1}$, we need only solve the following inequalities:

$$
\begin{aligned}
& K \geq \frac{\gamma}{2}+\frac{\varepsilon \beta}{2}, \\
& K \geq \frac{1}{4 \gamma}+\frac{\varepsilon}{4}, \\
& K \geq \frac{\varepsilon}{2 \beta}+\frac{1}{2 \varepsilon}+\varepsilon .
\end{aligned}
$$

Among all such constants satisfying the constraints (3.11)-(3.13), we desire the choice that minimizes $K$ and so, by (3.7), maximizes the estimates decay rate of $E_{2}(z)$. Since, from (3.13), a lower bound for $K$ is

$$
K \geq \frac{\varepsilon}{2 \beta}+\left(\sqrt{\varepsilon}-\frac{1}{\sqrt{2 \varepsilon}}\right)^{2}+\sqrt{2}>\sqrt{2},
$$

the smallest value of $K$ will be not less than $\sqrt{2}$. If we make the choice

$$
\varepsilon=\frac{1}{\sqrt{2}}, \quad \beta=\frac{1}{4}, \quad \gamma=\frac{1}{5},
$$

then it is easily seen that

$$
K=\sqrt{2}+\frac{1}{10}=1.514
$$

Hence, from (3.7), we conclude that $E_{2}(z)$ satisfies the differential inequality

$$
E_{2}(z)+1.514 \lambda_{1}^{-1 / 2} E_{2}^{\prime}(z) \leq 0, \quad z \geq 0 .
$$

Inequality (3.5) follows by integrating (3.14). To establish (3.6), we recall (3.3); it follows that

$$
-\frac{1}{2} \frac{d}{d z} \int_{D}\left(\phi_{, \alpha \beta} \phi_{, \alpha \beta}+\phi_{, 33}^{2}\right) d A \leq E_{2}(0) e^{-0.66 \sqrt{\lambda_{1}} z} .
$$

Now integrate both sides of (3.15) from $z$ to $\infty$ to get

$$
\int_{D_{z}}\left(\phi_{, \alpha \beta} \phi_{, \alpha \beta}+\phi_{, 33}^{2}\right) d A \leq 3.028 \lambda_{1}^{-1 / 2} E_{2}(0) e^{-0.66 \sqrt{\lambda_{1} z}} .
$$

We now introduce the notation

$$
E_{3}(z)=\int_{R_{z}}\left|\Delta \phi_{, 3}\right|^{2} d V=\int_{R_{z}} \phi_{, i i 3} \phi_{, j j 3} d A, \quad z \geq 0,
$$

and note that

$$
\int_{D_{z}} \phi_{, \alpha \beta} \phi_{, \alpha \beta} d A=\int_{D_{z}} \phi_{, \alpha \alpha} \phi_{, \beta \beta} d A
$$

and

$$
\begin{aligned}
E_{2}(z)-E_{3}(z) & =2 \int_{R_{z}} \phi_{, \alpha 33} \phi_{, \alpha 33} d V-2 \int_{R_{z}} \phi_{, \alpha \alpha 3} \phi_{, \alpha \alpha 3} d V \\
& =2 \int_{R_{z}}\left(\phi_{, \alpha 3} \phi_{, \alpha 33}\right)_{, 3} d V-2 \int_{R_{z}} \phi_{, \alpha 3} \phi_{, \alpha 333} d V+2 \int_{R_{z}} \phi_{, \alpha 3} \phi_{, \alpha 333} d V \\
& =-\frac{d}{d z} \int_{D_{z}} \phi_{, \alpha 3} \phi_{, \alpha 3} d A
\end{aligned}
$$


Hence, we obtain

$$
-\frac{d}{d z} \int_{D_{z}} \phi_{, \alpha 3} \phi_{, \alpha 3} d A \leq E_{2}(z) \leq E_{2}(0) e^{-0.66 \sqrt{\lambda_{1}} z} .
$$

An integration of (3.17) from $z$ to $\infty$ yields

$$
2 \int_{D_{z}} \phi_{, \alpha 3} \phi_{, \alpha 3} d A \leq 3.028 \lambda_{1}^{-1 / 2} E_{2}(0) e^{-0.66 \sqrt{\lambda_{1}} z} .
$$

Combining (3.16) and (3.18), we obtain the cross-sectional energy integral estimate

$$
\int_{D_{z}}\left(\phi_{, \alpha \beta} \phi_{, \alpha \beta}+2 \phi_{, \alpha 3} \phi_{, \alpha 3}+\phi_{, 33}^{2}\right) d A \leq 6.056 \lambda_{1}^{-1 / 2} E_{2}(0) e^{-0.66 \sqrt{\lambda_{1} z}} .
$$

Integrating (3.19), we arrive finally at

$$
E_{1}(z) \leq \frac{9.17}{\lambda_{1}} E_{2}(0) e^{-0.66 \sqrt{\lambda_{1}} z}, \quad z \geq 0
$$

which is (3.6).

4. An alternative energy estimate. In [7] the authors introduced a weighted energy integral in treating a similar problem for the Navier-Stokes equations. It has some advantages particularly in dealing with more complicated equations. We make use of this technique in this section to derive alternative energy estimates.

We first define the weighted energy

$$
\widetilde{E}_{\alpha}(z)=\int_{z}^{\infty} \int_{D_{\xi}}(\xi-z)^{\alpha+2} \phi_{, i j} \phi_{, i j} d V, \quad z \geq 0,
$$

where $\alpha$ is a positive parameter. Since we already know that $E_{1}(z)$ decays exponentially, we know that this weighted integral exists.

Obviously, we have

$$
\widetilde{E}_{\alpha}^{\prime}(z)=-(2+\alpha) \int_{R_{z}}(\xi-z)^{\alpha+1} \phi_{, i j} \phi_{, i j} d V .
$$

Now, we derive the decay estimates for $\widetilde{E}_{\alpha}(z)$ :

$$
\begin{aligned}
\widetilde{E}_{\alpha}(z)= & -\int_{R_{z}}(\xi-z)^{\alpha+2} \phi_{, i j j} \phi_{, i} d V-(2+\alpha) \int_{R_{z}}(\xi-z)^{\alpha+1} \phi_{, i 3} \phi_{, i} d V \\
= & -(2+\alpha) \int_{R_{z}}(\xi-z)^{\alpha+1} \phi_{, i 3} \phi_{, i} d V+(2+\alpha) \int_{R_{z}}(\xi-z)^{\alpha+1} \phi_{, 3 j j} \phi d V \\
= & -2(2+\alpha) \int_{R_{z}}(\xi-z)^{\alpha+1} \phi_{, i 3} \phi_{, i} d V \\
& -(2+\alpha)(1+\alpha) \int_{R_{z}}(\xi-z)^{\alpha} \phi_{, 33} \phi d V \\
= & I_{1}+I_{2} .
\end{aligned}
$$


Now

$$
\begin{aligned}
I_{2} & \leq(2+\alpha)(1+\alpha)\left[\int_{R_{x}}(\xi-z)^{\alpha+1} \phi_{, 33}^{2} d V \cdot \int_{R_{z}}(\xi-z)^{\alpha-1} \phi^{2} d V\right]^{1 / 2} \\
& \leq \frac{(2+\alpha)(1+\alpha)}{\sqrt{\lambda_{1}}}\left[\int_{R_{z}}(\xi-z)^{\alpha+1} \phi_{, 33}^{2} d V \int_{R_{z}}(\xi-z)^{\alpha-1} \phi_{, \alpha} \phi_{, \alpha} d V\right]^{1 / 2} .
\end{aligned}
$$

On the other hand, we have

$$
\begin{aligned}
\int_{R_{z}}(\xi & -z)^{\alpha-1} \phi_{, \alpha} \phi_{, \alpha} d V \\
= & \frac{1}{\alpha} \int_{R_{z}}\left[(\xi-z)^{\alpha} \phi_{, \alpha} \phi_{, \alpha}\right]_{, 3} d V \\
& -\frac{2}{\alpha} \int_{R_{z}}(\xi-z)^{\alpha} \phi_{, \alpha} \phi_{, \alpha 3} d V \\
\leq & \frac{2}{\alpha}\left[\int_{R_{z}}(\xi-z)^{\alpha-1} \phi_{, \alpha} \phi_{, \alpha} d V \cdot \int_{R_{z}}(\xi-z)^{\alpha+1} \phi_{, \alpha 3} \phi_{, \alpha 3} d V\right]^{1 / 2} .
\end{aligned}
$$

Combining (4.4) and (4.5), we obtain

$$
\begin{aligned}
I_{2} \leq & \frac{2(2+\alpha)(1+\alpha)}{\alpha \sqrt{\lambda_{1}}}\left(\int_{R_{z}}(\xi-z)^{\alpha+1} \phi_{, 33}^{2} d V\right)^{1 / 2} \\
& \times\left(\int_{R_{z}}(\xi-z)^{\alpha+1} \phi_{, \alpha 3} \phi_{, \alpha 3} d V\right)^{1 / 2}
\end{aligned}
$$

For $I_{1}$, upon using Schwarz's inequality, we have

$$
\begin{aligned}
I_{1} \leq & 2(2+\alpha)\left[\int_{R_{z}}(\xi-z)^{\alpha+1} \phi_{, \alpha 3} \phi_{, \alpha 3} d V \cdot \int_{R_{z}}(\xi-z)^{\alpha+1} \phi_{, \alpha} \phi_{, \alpha} d V\right]^{1 / 2} \\
& +2(2+\alpha)\left[\int_{R_{z}}(\xi-z)^{\alpha+1} \phi_{, 33}^{2} d V \cdot \int_{R_{z}}(\xi-z)^{\alpha+1} \phi_{, 3}^{2} d V\right]^{1 / 2} \\
\leq & \frac{2(2+\alpha)}{\sqrt{\Lambda}}\left[\int_{R_{z}}(\xi-z)^{\alpha+1} \phi_{, \alpha 3} \phi_{, \alpha 3} d V \cdot \int_{R_{z}}(\xi-z)^{\alpha+1} \phi_{, \alpha \beta} \phi_{, \alpha \beta} d V\right]^{1 / 2} \\
& +\frac{2(2+\alpha)}{\sqrt{\lambda_{1}}}\left[\int_{R_{z}}(\xi-z)^{\alpha+1} \phi_{,, 33}^{2} d V \cdot \int_{R_{z}}(\xi-z)^{\alpha+1} \phi_{, \alpha 3} \phi_{, \alpha 3} d V\right]^{1 / 2}
\end{aligned}
$$

Combining (4.3), (4.6), and (4.7) and applying the arithmetic geometric mean in- 
equality, it follows that

$$
\begin{aligned}
\widetilde{E}_{\alpha}(z) \leq & \frac{2(2+\alpha)(1+2 \alpha)}{\alpha \sqrt{\lambda_{1}}}\left[\int_{R_{z}}(\xi-z)^{1+\alpha} \phi_{, 33}^{2} d V \cdot \int_{R_{z}}(\xi-z)^{\alpha+1} \phi_{, \alpha 3} \phi_{\alpha 3} d V\right]^{1 / 2} \\
& +\frac{2(2+\alpha)}{\sqrt{\Lambda}}\left[\int_{R_{z}}(\xi-z)^{\alpha+1} \phi_{, \alpha 3} \phi_{, \alpha 3} d V \cdot \int_{R_{z}}(\xi-z)^{\alpha+1} \phi_{, \alpha \beta} \phi_{, \alpha \beta} d V\right]^{1 / 2} \\
\leq & (2+\alpha)\left[\frac{(1+2 \alpha) \varepsilon}{\alpha \sqrt{\lambda_{1}}} \int_{R_{z}}(\xi-z)^{\alpha+1} \phi_{, 33}^{2} d V\right. \\
& \quad+\frac{(1+2 \alpha)}{\alpha \sqrt{\lambda_{1}} \varepsilon} \int_{R_{z}}(\xi-z)^{\alpha+1} \phi_{, \alpha 3} \phi_{, \alpha 3} d V \\
& \left.+\frac{\sigma}{\sqrt{\Lambda}} \int_{R_{z}}(\xi-z)^{\alpha+1} \phi_{, \alpha 3} \phi_{, \alpha 3} d V+\frac{1}{\sqrt{\Lambda} \sigma} \int_{R_{z}}(\xi-z)^{\alpha+1} \phi_{, \alpha \beta} \phi_{, \alpha \beta} d V\right] .
\end{aligned}
$$

Now, choose appropriate positive numbers $\varepsilon$ and $\sigma$ such that

$$
\begin{gathered}
\frac{(1+2 \alpha) \varepsilon}{\alpha \sqrt{\lambda_{1}}}=\frac{1}{\sqrt{\Lambda} \sigma}, \\
\frac{1+2 \alpha}{\alpha \sqrt{\lambda_{1}} \varepsilon}+\frac{\sigma}{\sqrt{\Lambda}}=\frac{2}{\sqrt{\Lambda} \sigma} .
\end{gathered}
$$

Solving the algebraic equations (5.9), (5.10), we get

$$
\sigma=\sqrt{2 \lambda_{1}} /\left[((1+2 \alpha) / \alpha)^{2} \Lambda+\lambda_{1}\right]^{1 / 2} .
$$

Then (4.8) implies

$$
\widetilde{E}_{\alpha}(z) \leq-\frac{1}{\sqrt{\Lambda} \sigma} \widetilde{E}_{\alpha}^{\prime}(z), \quad z \geq 0 .
$$

Integrating (4.12), we obtain

$$
\widetilde{E}_{\alpha}(z) \leq \widetilde{E}_{\alpha}(0) e^{-\sqrt{\Lambda} \sigma z}=\widetilde{E}_{\alpha}(0) e^{-K_{1} z}
$$

where

$$
K_{1}=\sqrt{2 \lambda_{1}} / \sqrt{((1+2 \alpha) / \alpha)^{2}+\lambda_{1} / \Lambda} .
$$

When $\alpha=1$, we have

$$
\widetilde{E}_{1}(z) \leq \widetilde{E}_{1}(0) e^{-\sqrt{2 \lambda_{1}} z / \sqrt{9+\lambda_{1} / \Lambda}}
$$

For $\alpha \gg 1$, we obtain

$$
\widetilde{E}_{\alpha}(z) \leq \widetilde{E}_{\alpha}(0) e^{-\sqrt{2 \lambda_{1}} z / \sqrt{4+\varepsilon+\lambda_{1} / \Lambda}},
$$

where $\varepsilon$ is an arbitrary small positive number. 
Therefore, we have

THeOREM 4.1. Suppose $\phi$ is a solution of the system (2.1)-(2.5). Let $\alpha$ be any positive number; then the energy $\widetilde{E}_{\alpha}(z)$ satisfies the inequality

$$
\widetilde{E}_{\alpha}(z) \leq \widetilde{E}_{\alpha}(0) e^{-K_{1} z}, \quad z \geq 0,
$$

where $K_{1}$ is defined by (4.14).

Specifically, if $\alpha=1$, then the energy $\widetilde{E}_{\alpha}(z)$ satisfies

$$
\widetilde{E}_{1}(z) \leq \widetilde{E}_{1}(0) e^{-\sqrt{2 \lambda_{1}} z / \sqrt{9+\lambda_{1} / \Lambda}}
$$

Since in this method the number of dimensions comes into the estimates only through the eigenvalues $\lambda_{1}$ and $\Lambda$, if we consider the analogous problem in $\mathbb{R}^{2}$ where $\Lambda=4 \lambda_{1}$, we find in this case

$$
K_{1}=\frac{\sqrt{2 \lambda_{1}}}{\sqrt{((1+2 \alpha) / \alpha)^{2}+\frac{1}{4}}} \leq \frac{2 \sqrt{2 \lambda_{1}}}{\sqrt{17}}=\frac{2 \sqrt{2}}{\sqrt{17}} \frac{\pi}{h}
$$

where $h$ is the width of the member. Thus for $\alpha$ large the decay rate $K_{1}$ approaches $2 \sqrt{2 / 17} \pi h^{-1}$. But it is not as sharp as those found by Knowles [1] or Horgan [3].

5. The equivalence and bounds of energies. In [1] Knowles introduced the "higherorder energy" $E_{2}(z)$ for the biharmonic equation in a semi-infinite strip. His aim was to make use of a judicious combination of $E_{1}(z)$ and $E_{2}(z)$ in order to improve the energy decay rate. It is easy to show that $E_{1}(z)$ and $E_{2}(z)$ have the same decay rate, since there exists an equivalence between $E_{1}(z)$ and $E_{2}(z)$.

Lemma 5.1. Let $\phi$ be a solution of the system (2.1)-(2.5); then for any $z \geq 0$, the following inequality holds:

$$
\int_{R_{z}} \phi_{, \alpha 3} \phi_{, \alpha 3} d V \leq \frac{3}{2} \int_{R_{z}}\left(\phi_{, \alpha \beta} \phi_{, \alpha \beta}+\phi_{, 33}^{2}\right) d V .
$$

Proof. It is clear that

$$
\begin{aligned}
\int_{R_{z}} \phi_{, i j} \phi_{, i j} d V & =-\int_{R_{z}}(\xi-z)\left(\phi_{, i j} \phi_{, i j}\right)_{, 3} d V \\
& =-2 \int_{R_{z}}(\xi-z) \phi_{, i j} \phi_{, i j 3} d V \\
& \leq \frac{1}{2} \int_{R_{z}} \phi_{, i j} \phi_{, i j} d V+2 \int_{R_{z}}(\xi-z)^{2} \phi_{, i j 3} \phi_{, i j 3} d V .
\end{aligned}
$$


Hence, we get

$$
\begin{aligned}
& \int_{R_{z}} \phi_{, i j} \phi_{, i j} d V \\
& \leq 4 \int_{R_{z}}(\xi-z)^{2} \phi_{, i j 3} \phi_{, i j 3} d V \\
&=-4 \int_{R_{z}}(\xi-z)^{2} \phi_{, i j j 3} \phi_{, i 3} d V-8 \int_{R_{z}}(\xi-z) \phi_{, i 33} \phi_{, i 3} d V \\
&= 8 \int_{R_{z}}(\xi-z) \phi_{, j j 33} \phi_{, 3} d V-4 \int_{R_{z}}(\xi-z)\left(\phi_{, i 3} \phi_{, i 3}\right)_{, 3} d V \\
&=-4 \int_{R_{z}}(\xi-z)\left(\phi_{, \alpha \beta} \phi_{, \alpha \beta}\right)_{3} d V+4 \int_{R_{z}}(\xi-z)\left(\phi_{, \alpha 3} \phi_{, \alpha 3}\right) d V \\
&+4 \int_{R_{z}} \phi_{, i 3} \phi_{, i 3} d V \\
&= 4 \int_{R_{z}}\left(\phi_{, \alpha \beta} \phi_{, \alpha \beta}+\phi_{, 33}^{2}\right) d V,
\end{aligned}
$$

and Lemma 5.1 follows directly.

THEOREM 5.2. $E_{1}(z)$ and $E_{2}(z)$ satisfy the following inequalities for arbitrary $z_{0}>$ 0 :

$$
\begin{aligned}
& E_{1}(z) \leq 147 \lambda_{1}^{-1} E_{2}(z), \\
& z_{0}^{2} E_{2}\left(z+z_{0}\right)<E_{1}(z) .
\end{aligned}
$$

Proof. First, we prove (5.2).

By (3.5) of Theorem 3.1, we have the inequality

$$
E_{2}(z) \leq-1.514 \lambda_{1}^{-1 / 2} E_{2}^{\prime}(z), \quad z \geq 0 .
$$

The above inequality may be rewritten in the form

$$
-\frac{1}{2} \frac{d}{d z} \int_{D_{z}}\left(\phi_{, \alpha \beta} \phi_{, \alpha \beta}+\phi_{, 33}^{2}\right) d A \leq-1.514 \lambda_{1}^{-1 / 2} E_{2}^{\prime}(z) .
$$

If we integrate both sides of (5.4) from $z$ to $\infty$, it follows that

$$
\int_{D_{z}}\left(\phi_{, \alpha \beta} \phi_{, \alpha \beta}+\phi_{, 33}^{2}\right) d A \leq 3.028 \lambda_{1}^{-1 / 2} E_{2}(z) .
$$

Integrating (5.5) once again, we get

$$
\int_{R_{z}}\left(\phi_{, \alpha \beta} \phi_{, \alpha \beta}+\phi_{, 33}^{2}\right) d V \leq 3.028 \lambda_{1}^{-1 / 2} \int_{z}^{\infty} E_{2}(\xi) d \xi .
$$


If we make use of Lemma 5.1 and the above inequality, we obtain

$$
\begin{aligned}
E_{1}(z) & \leq 4 \int_{R_{z}}\left(\phi_{, \alpha \beta} \phi_{, \alpha \beta}+\phi_{, 33}^{2}\right) d V \\
& \leq 12.112 \lambda_{1}^{-1 / 2} \int_{z}^{\infty} E_{2}(\xi) d \xi \\
& =6.056 \lambda_{1}^{-1 / 2} \int_{D_{z}}\left(\phi_{, \alpha \beta} \phi_{, \alpha \beta}+\phi_{, 33}^{2}\right) d A \\
& =-6.056 \lambda_{1}^{-1 / 2} \int_{R_{z}}\left(\phi_{, \alpha \beta} \phi_{, \alpha \beta}+\phi_{, 33}^{2}\right)_{, 3} d V \\
& =-12.112 \lambda_{1}^{-1 / 2} \int_{R_{z}}\left(\phi_{, \alpha \beta} \phi_{, \alpha \beta 3}+\phi_{, 33} \phi_{, 333}\right) d V \\
& \leq 6.056 \frac{\lambda_{1}^{-1 / 2}}{\varepsilon} \int_{R_{z}} \phi_{, i j} \phi_{, i j} d V+6.056 \lambda_{1}^{-1 / 2} \varepsilon \int_{R_{z}} \phi_{, i j 3} \phi_{, i j 3} d V
\end{aligned}
$$

for arbitrary positive $\varepsilon$. If we choose $\varepsilon=12.112 \lambda_{1}^{-1 / 2}$, then (5.2) follows.

Now, we prove (5.3). It is clear that

$$
\begin{aligned}
0= & \int_{R_{z}}(\xi-z)^{2} \Delta \Delta \phi \phi_{, 33} d V \\
= & \int_{R_{z}}\left((\xi-z)^{2} \phi_{, i i j} \phi_{, 33}\right)_{, j} d V-\int_{R_{z}}(\xi-z)^{2} \phi_{, i i j} \phi_{, 33 j} d V \\
& -2 \int_{R_{z}}(\xi-z) \phi_{, i i 3} \phi_{, 33} d V \\
= & \int_{R_{z}}(\xi-z)^{2} \phi_{, i j} \phi_{, i j 33} d V+2 \int_{R_{z}}(\xi-z) \phi_{, 3 j} \phi_{, 33 j} d V \\
& +2 \int_{R_{z}}(\xi-z) \phi_{, i 3} \phi_{, i 33} d V+2 \int_{R_{z}} \phi_{, 33} \phi_{, 33} d V \\
= & -\int_{R_{z}}(\xi-z)^{2} \phi_{, i j 3} \phi_{, i j 3} d V-2 \int_{R_{z}}(\xi-z) \phi_{, i j} \phi_{, i j 3} d V \\
& -2 \int_{R_{z}} \phi_{, i 3} \phi_{, i 3} d V+2 \int_{R_{z}} \phi_{, 33} \phi_{, 33} d V \\
= & -\int_{R_{z}}(\xi-z)^{2} \phi_{, i j 3} \phi_{, i j 3} d V+\int_{R_{z}} \phi_{, i j} \phi_{, i j} d V \\
& -2 \int_{R_{z}} \phi_{, i 3} \phi_{, i 3} d V+2 \int_{R_{z}} \phi_{, 33}^{2} d V .
\end{aligned}
$$

The above equality reduces to

$$
\begin{aligned}
\int_{R_{z}} \phi_{, i j} \phi_{, i j} d V-2 \int_{R_{z}} \phi_{, \alpha 3} \phi_{, \alpha 3} d V & =\int_{R_{z}}(\xi-z)^{2} \phi_{, i j 3} \phi_{, i j 3} d V \\
& >\int_{R_{z+z_{0}}}(\xi-z)^{2} \phi_{, i j 3} \phi_{, i j 3} d V \\
& \geq z_{0}^{2} E_{2}\left(z+z_{0}\right) .
\end{aligned}
$$

This completes the proof of Theorem 5.2. 
For the weighted energy integral, we also have

THEOREM 5.3. $\widetilde{E}_{1}(z)$ and $E_{1}(z)$ satisfy the relation

$$
\widetilde{E}_{1}(z) \leq\left(\frac{24 \sqrt{3}}{\lambda_{1} \sqrt{\lambda_{1}}}+\frac{3 \sqrt{2}}{\sqrt{\lambda_{1} \Omega}}\right) E_{1}(z), \quad z \geq 0 .
$$

Proof.

$$
\begin{aligned}
\widetilde{E}_{1}(z) & =\int_{R_{z}}(\xi-z)^{3} \phi_{, i j} \phi_{, i j} d V \\
& =-\int_{R_{z}}(\xi-z)^{3} \phi_{, i j j} \phi_{, i} d V-3 \int_{R_{z}}(\xi-z)^{2} \phi_{, i 3} \phi_{, i} d V \\
& =3 \int_{R_{z}}(\xi-z)^{2} \phi_{, 3 j j} \phi d V-3 \int_{R_{z}}(\xi-z)^{2} \phi_{, i 3} \phi_{, i} d V \\
& =-6 \int_{R_{z}}(\xi-z) \phi_{, 33} \phi d V-6 \int_{R_{z}}(\xi-z)^{2} \phi_{, i 3} \phi_{, i} d V \\
& =12 \int_{R_{z}}(\xi-z) \phi_{, 3} \phi_{, 3} d V+6 \int_{R_{z}}(\xi-z) \phi_{, \alpha} \phi_{, \alpha} d V+6 \int_{R_{z}} \phi \phi{ }_{, 3} d V \\
& =I_{1}+I_{2}+I_{3} .
\end{aligned}
$$

For $I_{1}$, apply the Hölder inequality and Young's inequality $a b \leq \varepsilon a^{p}+\varepsilon^{-q / p} b^{q}$ to get

$$
\begin{aligned}
I_{1} & \leq 12\left(\int_{R_{z}}(\xi-z)^{3} \phi_{, 3} \phi_{, 3} d V\right)^{1 / 3}\left(\int_{R_{z}} \phi_{, 3} \phi_{, 3} d V\right)^{2 / 3} \\
& \leq 12 \varepsilon \int_{R_{z}}(\xi-z)^{3} \phi_{, 3} \phi_{, 3} d V+\frac{12}{\sqrt{\varepsilon}} \int_{R_{z}} \phi_{, 3} \phi_{, 3} d V \\
& \leq \frac{12 \varepsilon}{\lambda_{1}} \int_{R_{z}}(\xi-z)^{3} \phi_{, 3 \alpha} \phi_{, 3 \alpha} d V+\frac{12}{\sqrt{\varepsilon} \lambda_{1}} \int_{R_{z}} \phi_{, 3 \alpha} \phi_{, 3 \alpha} d V .
\end{aligned}
$$

Similarly, for $I_{2}$, we have

$$
\begin{aligned}
I_{2} & \leq 6\left(\int_{R_{z}}(\xi-z)^{3} \phi_{, \alpha} \phi_{, \alpha} d V\right)^{1 / 3}\left(\int_{R_{z}} \phi_{, \alpha} \phi_{, \alpha} d V\right)^{2 / 3} \\
& \leq \frac{6 \sigma}{\Lambda} \int_{R_{z}}(\xi-z)^{3} \phi_{, \alpha \beta} \phi_{, \alpha \beta} d V+\frac{6}{\sqrt{\sigma} \Lambda} \int_{R_{z}} \phi_{, \alpha \beta} \phi_{, \alpha \beta} d V .
\end{aligned}
$$

Take $\varepsilon=\lambda_{1} / 12$ and $\sigma=\Lambda / 12$, since $\lambda_{1} \leq \Lambda$; combining (5.7)-(5.9), we get

$$
\begin{aligned}
\widetilde{E}_{1}(z) \leq & \frac{1}{2} \int_{R_{z}}(\xi-z)^{3}\left(\phi_{, \alpha \beta} \phi_{, \alpha \beta}+2 \phi_{, \alpha 3} \phi_{, \alpha 3}\right) d V \\
& +\frac{24 \sqrt{3}}{\lambda_{1} \sqrt{\lambda_{1}}} \int_{R_{z}} \phi_{, \alpha 3} \phi_{, \alpha 3} d V+\frac{12 \sqrt{3}}{\lambda_{1} \sqrt{\lambda_{1}}} \int_{R_{z}} \phi_{, \alpha \beta} \phi_{, \alpha \beta} d V+6 \int_{R_{z}} \phi \phi_{, 3} d V \\
\leq & \frac{1}{2} \widetilde{E}_{1}(z)+\frac{12 \sqrt{3}}{\lambda_{1} \sqrt{\lambda_{1}}} E_{1}(z)+6 \int_{R_{z}} \phi \phi_{, 3} d V .
\end{aligned}
$$


Hence, we obtain

$$
\begin{aligned}
\widetilde{E}_{1}(z) & \leq \frac{24 \sqrt{3}}{\lambda_{1} \sqrt{\lambda_{1}}} E_{1}(z)+12 \int_{R_{z}} \phi \phi_{, 3} d V \\
& \leq \frac{24 \sqrt{3}}{\lambda_{1} \sqrt{\lambda_{1}}} E_{1}(z)+6 \tau \int_{R_{z}} \phi_{, 3}^{2} d V+\frac{6}{\tau} \int_{R_{z}} \phi^{2} d V \\
& \leq \frac{24 \sqrt{3}}{\lambda_{1} \sqrt{\lambda_{1}}} E_{1}(z)+\frac{6 \tau}{\lambda_{1}} \int_{R_{z}} \phi_{, 3 \alpha} \phi_{, 3 \alpha} d V+\frac{6}{\tau \Omega} \int_{R_{z}} \phi_{, \alpha \beta} \phi_{, \alpha \beta} d V .
\end{aligned}
$$

Now, choose $\tau>0$ such that

$$
\frac{\tau}{\lambda_{1}}=\frac{2}{\tau \Omega} \quad \text { or } \quad \tau=\sqrt{\frac{2 \lambda_{1}}{\Omega}} .
$$

Finally, we arrive at the conclusion of Theorem 5.3.

With Theorems 5.2 and 5.3 in mind, we know that either $E_{2}(z)$ or $\widetilde{E}_{1}(z)$ can be bounded by $E_{1}(z)$, so what we need to derive is an upper bound for energy $E_{1}(0)$ only. We have

TheOREM 5.4. The total $E_{1}(0)$ can be bounded in terms of boundary data, i.e., $E_{1}(0)$ satisfies the inequality

$$
\begin{aligned}
E_{1}(0) \leq & \frac{1}{4} \int_{D}\left(5 f_{, \alpha \beta} f_{, \alpha \beta}+4 f_{, \alpha \beta} g_{, \alpha \beta}+g_{, \alpha \beta} g_{, \alpha \beta}\right) d A \\
& +\frac{1}{4} \int_{D}\left(3 g_{, \alpha} g_{, \alpha}+f_{, \alpha} f_{, \alpha}\right) d A+\frac{1}{4} \int_{D}\left(5 g^{2}+4 g f+f^{2}\right) d A .
\end{aligned}
$$

Proof. Suppose $\psi$ is a sufficiently smooth function satisfying the boundary conditions:

$$
\begin{gathered}
\psi=\phi ; \quad \psi_{, 3}=\phi_{, 3} \quad \text { in } D_{0} ; \\
\psi=\frac{\partial \psi}{\partial n}=0 \quad \text { on } \partial D ; \\
\left.\psi_{, 3}, \psi_{, \alpha \beta} \rightarrow 0 \quad \text { (uniformly in }\left(x_{1}, x_{2}\right) \in D\right) \text { as } x_{3} \rightarrow \infty .
\end{gathered}
$$

Upon using Schwarz's inequality, we get

$$
\begin{aligned}
\left(\int_{R_{0}} \phi_{, i j} \psi_{, i j} d V\right)^{2} & \leq \int_{R_{0}} \phi_{, i j} \phi_{, i j} d V \cdot \int_{R_{0}} \psi_{, i j} \psi_{, i j} d V \\
& =E_{1}(0) \int_{R_{0}} \psi_{, i j} \psi_{, i j} d V .
\end{aligned}
$$

On the other hand, we note that

$$
E_{1}(0)=-\int_{D_{0}} \phi_{, i} \phi_{, i 3} d A+\int_{D_{0}} \phi_{, i i 3} \phi d A .
$$


But

$$
\begin{aligned}
\int_{R_{0}} \phi_{, i j} \psi_{, i j} d V & =\int_{R_{0}}\left(\phi_{, i j} \psi_{, i}\right)_{, j} d V-\int_{R_{0}} \phi_{, i j j} \psi_{, i} d V \\
& =-\int_{D_{0}} \phi_{, i 3} \psi_{, i} d A+\int_{D_{0}} \phi_{, 3 j j} \psi d A \\
& =-\int_{D_{0}} \phi_{, i 3} \phi_{, i} d A+\int_{D_{0}} \phi_{, 3 j j} \phi d A \\
& =E_{1}(0) .
\end{aligned}
$$

Hence, we have

$$
E_{1}(0) \leq \int_{R_{0}} \psi_{, i j} \psi_{, i j} d V
$$

We set

$$
\psi\left(x_{1}, x_{2}, x_{3}\right)=\left(f+x_{3} g+r x_{3} f\right) e^{-r x_{3}}
$$

where $r$ is a positive constant to be determined later.

It is easy to verify that $\psi$ satisfies all of the boundary conditions $(5.10)-(5.12)$. It remains to calculate an upper bound for $E_{1}(0)$. By calculating, we obtain

$$
\begin{aligned}
E_{1}(0) \leq & \int_{0}^{\infty} \int_{D_{\xi}} \psi_{, i j} \psi_{, i j} d V \\
= & \frac{1}{2 r} \int_{D}\left[f_{, \alpha \beta} f_{, \alpha \beta}+2 g_{, \alpha} g_{, \alpha}+r^{2}(2 g+r f)^{2}\right] d A \\
& +\frac{1}{2 r^{2}} \int_{D}\left[f_{, \alpha \beta}\left(g_{, \alpha \beta}+r f_{, \alpha \beta}\right)-r g_{, \alpha}\left(g_{, \alpha}+r f_{, \alpha}\right)\right. \\
& +\frac{1}{4 r^{3}} \int_{D}\left[\left(g_{, \alpha \beta}+r f_{, \alpha \beta}\right)\left(g_{, \alpha \beta}+r f_{, \alpha \beta}\right)\right. \\
& \left.\quad+r^{2}\left(g_{, \alpha}+r f_{, \alpha}\right)(g+r f)\right] d A
\end{aligned}
$$

If we take $r=1$, we are led finally to the conclusion of Theorem 5.4.

Acknowledgment. This paper was written while the author held a visiting appointment at Cornell University. The author is grateful to Professor L. E. Payne for many helpful discussions concerning this research and for his reading of the final manuscript.

\section{REFERENCES}

[1] J. K. Knowles, An energy estimate for the biharmonic equation and its application to Saint-Venant's principle in plane elastostatistics, Indian J. Pure Appl. Math. 14, 791-805 (1983)

[2] J. K. Knowles, On Saint-Venant's principle in the two dimensional linear theory of elasticity, Arch. Rational Mech. Anal. 21, 1-22 (1966)

[3] C. O. Horgan, Decay estimates for the biharmonic equation with applications to Saint-Venant's principles in plane elasticity and Stokes flows, Quart. Appl. Math. 47, 147-157 (1989)

[4] C. O. Horgan and J. K. Knowles, Recent developments concerning Saint-Venant's principle, T. Y. Wu and J. W. Hutchinson (eds.), Adv. Appl. Mech. 23, Academic Press, San Diego, 1983, pp. 179-269 
[5] J. N. Flavin, On Knowles' version of Saint-Venant's principle in two dimensional elastostatics, Arch. Rational Mech. Anal. 53, 366-375 (1974)

[6] O. A. Oleinik and G. A. Yosifian, The Saint-Venant's principle in the two-dimensional theory of elasticity and boundary problems for a biharmonic equation in unbounded domains, Sibirsk. Math. Zh. 19, 1154-1165 (1978); English transl., Siberian Math. J. 19, 813-822 (1978)

[7] K. A. Ames and L. E. Payne, Decay estimates in steady pipe flow, SIAM J. Math. Anal. 20, 789-815 (1989)

[8] G. Faber, Beweis, dass under allen homogenen membranen von gleicher Fläche und gleicher Spannung die kreisförmige den tiefsten Grundton gibt, Sitz Bayer Akad. Wiss., 169-192 (1923)

[9] G. Pólya and G. Szegö, Isoperimetric inequalities in mathematical physics, Ann. of Math. Stud. 27, Princeton Univ. Press, Princeton, NJ, 1951

[10] C. O. Horgan, Recent developments concerning Saint-Venant's principle: an update, Appl. Mech. Rev. 42, 295-303 (1989) 